\title{
Deep Learning Application for Vocal Fold Disease Prediction Through Voice Recognition: Preliminary Development Study
}

Hao-Chun $\mathrm{Hu}^{1,2,3}$, MD; Shyue-Yih Chang ${ }^{4}$, MD; Chuen-Heng Wang ${ }^{5}$, MSc; Kai-Jun Li ${ }^{2}, \mathrm{MSc}$; Hsiao-Yun Cho ${ }^{2,6}$, MD; Yi-Ting Chen ${ }^{5}$, MSc; Chang-Jung Lu ${ }^{4}$, MSc; Tzu-Pei Tsai ${ }^{4}$, MSc; Oscar Kuang-Sheng Lee ${ }^{1,7,8,9}$, MD, PhD

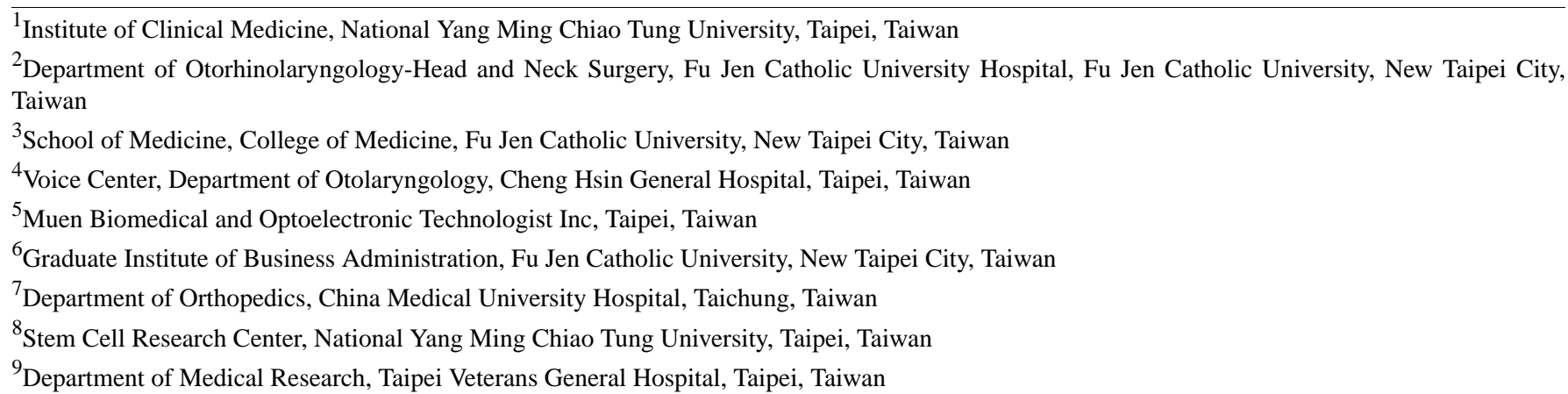

Corresponding Author:

Oscar Kuang-Sheng Lee, MD, PhD

Institute of Clinical Medicine

National Yang Ming Chiao Tung University

No 155, Section 2, Li-Nong Street, Beitou District

Taipei, 11221

Taiwan

Phone: 886228757391

Fax: 886228757841

Email: oscarlee9203@gmail.com

\section{Abstract}

Background: Dysphonia influences the quality of life by interfering with communication. However, a laryngoscopic examination is expensive and not readily accessible in primary care units. Experienced laryngologists are required to achieve an accurate diagnosis.

Objective: This study sought to detect various vocal fold diseases through pathological voice recognition using artificial intelligence.

Methods: We collected 189 normal voice samples and 552 samples of individuals with voice disorders, including vocal atrophy $(n=224)$, unilateral vocal paralysis $(n=50)$, organic vocal fold lesions $(n=248)$, and adductor spasmodic dysphonia $(n=30)$. The 741 samples were divided into 2 sets: 593 samples as the training set and 148 samples as the testing set. A convolutional neural network approach was applied to train the model, and findings were compared with those of human specialists.

Results: The convolutional neural network model achieved a sensitivity of 0.66 , a specificity of 0.91 , and an overall accuracy of $66.9 \%$ for distinguishing normal voice, vocal atrophy, unilateral vocal paralysis, organic vocal fold lesions, and adductor spasmodic dysphonia. Compared with the accuracy of human specialists, the overall accuracy rates were $60.1 \%$ and $56.1 \%$ for the 2 laryngologists and $51.4 \%$ and $43.2 \%$ for the 2 general ear, nose, and throat doctors.

Conclusions: Voice alone could be used for common vocal fold disease recognition through a deep learning approach after training with our Mandarin pathological voice database. This approach involving artificial intelligence could be clinically useful for screening general vocal fold disease using the voice. The approach includes a quick survey and a general health examination. It can be applied during telemedicine in areas with primary care units lacking laryngoscopic abilities. It could support physicians when prescreening cases by allowing for invasive examinations to be performed only for cases involving problems with automatic recognition or listening and for professional analyses of other clinical examination results that reveal doubts about the presence of pathologies. 
(J Med Internet Res 2021;23(6):e25247) doi: 10.2196/25247

\section{KEYWORDS}

artificial intelligence; convolutional neural network; dysphonia; pathological voice; vocal fold disease; voice pathology identification

\section{Introduction}

The impact of a voice disorder has been increasingly recognized as a public health concern. Dysphonia influences the quality of physical, social, and occupational aspects of life by interfering with communication [1]. A nationwide insurance claims data analysis of treatment seeking for dysphonia showed a prevalence rate of $0.98 \%$ among 55 million individuals [2], and this rate reached $2.5 \%$ among those older than 70 years [2]. However, the overall dysphonia incidence for the aging population is estimated to be much higher (12\%-35\%) [3], which may imply that dysphonia is commonly overlooked by patients, resulting in underdiagnosis.

According to the state-of-the-art clinical practice guidelines for dysphonia of the American Academy of Otolaryngology-Head and Neck Surgery Foundation, a laryngoscopic examination is recommended if dysphonia fails to resolve or improve within 4 weeks [4]. A comparison of diagnoses made by primary care physicians and those made by laryngologists and speech-language pathologists with experience in interpreting stroboscopy at multidisciplinary voice clinics indicated that the primary care physicians' diagnoses of dysphonia were different in $45 \%-70 \%$ of cases [4]. However, the laryngoscopic examination is an invasive procedure. To achieve an accurate diagnosis, it must be performed by an experienced laryngologist. The examination equipment is expensive and not generally available in primary care units. In places without sufficient medical resources, delayed diagnoses and treatments are common [5]. Therefore, a noninvasive diagnostic tool is needed to resolve this problem. Although this tool cannot replace the laryngoscopic examination by an experienced physician, it is worthwhile to develop because a noninvasive tool to screen significant clinical conditions could encourage patients to visit a voice clinic for further evaluation.

Several recent studies have attempted to distinguish normal and abnormal voices by using various machine learning-based classifiers that have the potential for detecting pathological voices [5-9]. To date, the highest accuracy of pathological voice detection achieved by using a deep neural network has been
99.32\% [5]. However, the differential diagnosis of various types of pathological voices has not been widely reported. The vibration patterns of vocal fold observed by high-speed video for common vocal fold diseases, including vocal atrophy, unilateral vocal paralysis, and organic vocal fold lesions, are completely different [10]. We hypothesized that different vibration patterns could result in different voice features. This study sought to detect various vocal fold diseases through pathological voice recognition using a deep learning approach.

\section{Methods}

\section{Sample Collection}

This study was performed following the principles expressed in the Declaration of Helsinki, and approved by the Institutional Ethics and Research Committee of Cheng Hsin General Hospital and Fu Jen Catholic University. Voice samples were obtained from the Voice Center of Chen Hsin General Hospital and the Department of Otorhinolaryngology-Head and Neck Surgery of Fu Jen Catholic University Hospital. These samples included 189 normal voice samples and 552 samples of voice disorders, including vocal atrophy $(n=224)$, unilateral vocal paralysis $(n=50)$, organic vocal fold lesions $(n=248)$, and adductor spasmodic dysphonia $(n=30)$. Voice samples of a sustained vowel sound /a:/ followed by continuous speech of a Mandarin passage [11] (Multimedia Appendix 1) were recorded at a comfortable loudness level with a microphone-to-mouth distance of approximately $15-20 \mathrm{~cm}$ using a high-quality microphone with a digital amplifier and a 40- to 45-dB background noise level. The sampling rate was $44,100 \mathrm{~Hz}$ with 16 -bit resolution, and data were saved in an uncompressed .wav format.

\section{Comparison and Evaluation}

We first divided the 741 samples into 2 sets: 593 samples for the training set and 148 samples for the testing set. Using computer-based randomization, we selected 152 of the 189 normal voice samples, 40 of the 50 unilateral vocal paralysis samples, 24 of the 30 adductor spasmodic dysphonia samples, 179 of the 224 vocal atrophy samples, and 198 of the 248 organic vocal fold lesion samples for the training set (Table 1).

Table 1. Details of the voice samples used for experiments $(\mathrm{N}=741)$.

\begin{tabular}{lll}
\hline Sample & Training set $(\mathrm{n}=593)$ & Test set $(\mathrm{n}=148)$ \\
\hline Normal & 152 & 37 \\
Unilateral vocal paralysis & 40 & 10 \\
Adductor spasmodic dysphonia & 24 & 6 \\
Vocal atrophy & 179 & 45 \\
Organic vocal fold lesions & 198 & 50 \\
\hline
\end{tabular}

To manage the limited size of the training set, we used a mix-up approach for data augmentation [12]. The mix-up approach has been applied for audio scene classification using convoluted neural networks (CNNs) to reduce overfitting and obtain higher prediction accuracy [13]. We randomly selected 2 voice files and mixed them into 1 voice file with randomly selected weights 
to construct the virtual training examples. Next, we randomly cropped each of these voice files to achieve 10 voice files with a length of 11.88 seconds (plateau point of the training length within the graphics processing unit memory limitations of our hardware, according to our preliminary tests). Additionally, we used oversampling to adjust the class distribution of the data [14].

A 2D graph is ideal for extracting features when using CNNs. Therefore, we performed Mel frequency cepstral coefficients (MFCCs) for the processed voice file to obtain a spectrogram. Feature extraction from MFCCs was performed using pre-emphasis, windowing, fast Fourier transform, Mel filtering, nonlinear transformation, and discrete cosine transform [15]. The first feature consisted of 40-dimension MFCCs [16,17]. Next, for the second and third features, we calculated the MFCC trajectories over time (delta MFCCs) and the second-order delta of MFCCs. Therefore, there were 3 channels of input features that could be considered a color image (ie, red-green-blue in the computer vision field).

CNNs have distinct feature representation-related characteristics, among which the lower layers provide general feature-extraction capabilities and the higher layers include information that is increasingly more specific to the original classification task [18]. This allows verbatim reuse of the generalized feature-extraction and representation of the lower CNN layers; the higher layers are fine-tuned toward secondary problem domains with characteristics related to the original. Therefore, instead of designing a new CNN with random parameter initialization, it is more suitable to adopt a pretrained $\mathrm{CNN}$ and fine-tune its parameterization toward specific classification domains. Spectrograms were quite different from normal images at first glance. However, the low-level features, including edges, corners, and shapes, were common in the normal images and spectrograms [19]. In a previous study, a spectrogram-based crowd sounds analysis using pretrained CNN models from the ImageNet data set showed great accuracy when distinguishing crowd emotions [19]. Another study also proved that pretrained $\mathrm{CNN}$ models yielded better performance than nontrained CNN models for classifying normal or pathological cases [18]. We used different CNN architectures, such as EfficientNet-B0 to B6 [20], SENet154 [21], Se_resnext101_32x4d [21], and se_resnet 152 [21] models, from the ImageNet data set that have been pretrained for transfer learning. We classified pathological conditions into 2 (normal voice; adductor spasmodic dysphonia plus organic vocal fold lesions plus unilateral vocal paralysis plus vocal atrophy), 3 (normal voice; adductor spasmodic dysphonia; organic vocal fold lesions plus unilateral vocal paralysis plus vocal atrophy), 4 (normal voice; adductor spasmodic dysphonia; organic vocal fold lesions; unilateral vocal paralysis plus vocal atrophy), or 5 (normal voice; adductor spasmodic dysphonia; organic vocal fold lesions; unilateral vocal paralysis; vocal atrophy) different conditions and trained the CNN. For the final prediction of an input instance, we used the maximum probability to obtain the label.

In terms of hyperparameter settings for fine-tuning among the training set, 474 of 593 samples (79.9\%) were used for initial training and 119 of 593 samples (20.1\%) were used for validation. We added the dropout function and different data augmentation methods to prevent the model from overfitting in our data set $[22,23]$. The dropout rate was set at $0.25-0.5$ for regularization. Then, we trained the model using minibatches of 32 that were selected based on memory consumption [24]. The learning rate was tuned based on cosine annealing and a $1-$ cycle policy strategy $[25,26]$. By using the cosine annealing schedule, the model repeatedly fitted the gradient to the local minimum. The network was trained end-to-end using the Adam optimization algorithm, and it optimized the cross-entropy as a loss function [27]. For different classification problems in the model head, we applied a SoftMax layer as an output layer for multiclass classification or a sigmoid layer for binary classification. Finally, we assembled the model by average output probability to receive more robust results to minimize the bias of prediction error to improve the prediction accuracy of the CNN models [25]. The machine learning process was performed using Python 3.8 (Python Software Foundation) and PyTorch 1.7.1 for Ubuntu 18.04 (Facebook's AI Research lab [FAIR]). Furthermore, we invited 2 laryngologists and 2 general ear, nose, and throat (ENT) physicians who could speak Mandarin to categorize the voice samples of the testing sets into 5 classifications. We compared their classifications with those of our model.

\section{Statistical Analysis}

The effectiveness of our model was evaluated by several metrics, including accuracy, sensitivity, specificity, F1 score, receiver-operating characteristic (ROC) curve, and area under the curve (AUC). All metrics were calculated using Python.

\section{Results}

Voice samples in this study were composed of a sustained vowel sound and a continuous essay speech. We first compared the results by training different parts: the vowel sound alone, the essay alone, and the whole voice sample (ie, the vowel sound and essay). Because the vowel sound and essay group (F1 score $=0.65)$ achieved better F1 scores than the vowel sound group $(\mathrm{F} 1$ score $=0.54)$ and the essay group $(\mathrm{F} 1$ score $=0.57)$, we applied whole voice samples during subsequent machine learning. Figure 1 shows the changes in the loss function value over the training and validation sets, which demonstrated that our model could converge after running the optimization for a number of epochs. 
Figure 1. Illustration of the changes of the loss function value over the training and validation sets.

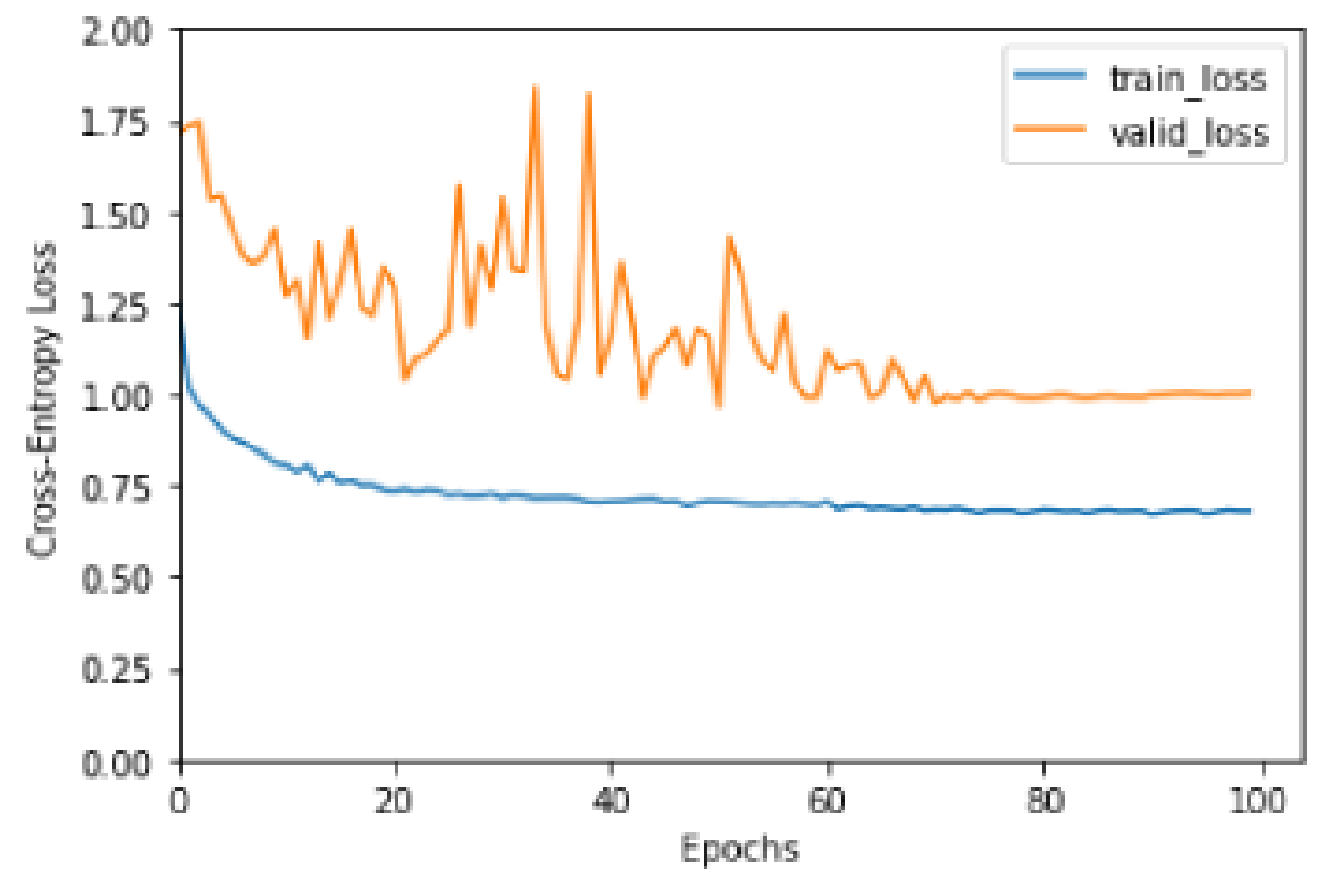

Table 2 presents the training results for the different classification methods, including 2 (normal voice; adductor spasmodic dysphonia plus organic vocal fold lesions plus unilateral vocal paralysis plus vocal atrophy), 3 (normal voice; adductor spasmodic dysphonia; organic vocal fold lesions plus unilateral vocal paralysis plus vocal atrophy), 4 (normal voice; adductor spasmodic dysphonia; organic vocal fold lesions; unilateral vocal paralysis; vocal atrophy), or 5 (normal voice; adductor spasmodic dysphonia; organic vocal fold lesions; unilateral vocal paralysis; vocal atrophy) different conditions trained by the CNN. The 2-classification condition could equally distinguish pathological voices from normal voices. In our model, the accuracy of pathological voice detection reached
95.3\%; the sensitivity was $99 \%$, specificity was $84 \%$, and AUC was 0.98 . Using the 3-classification condition, we aimed to identify adductor spasmodic dysphonia patients from those with other vocal fold pathologies. The accuracy was $91.2 \%$, sensitivity was $82 \%$, specificity was $93 \%$, and AUC was 0.91 . Using the 4-classification condition, vocal atrophy and unilateral vocal paralysis could be clinically grouped as "glottic insufficiency." For this condition, the accuracy was $71.0 \%$, sensitivity was $75 \%$, specificity was $89 \%$, and AUC was 0.88 . Using the 5-classification condition, the accuracy was $66.9 \%$, sensitivity was $66 \%$, specificity was $91 \%$, and AUC was 0.85 . Figure 2 shows the confusion matrix of these results. Figure 3 shows the ROC curves of these results.

Table 2. Performance of the artificial intelligence model for classifying voice disorders under different classification conditions.

\begin{tabular}{llllll}
\hline Class & Sensitivity & Specificity & Accuracy, $\%$ & F1 score & Average area under the curve values \\
\hline 2 & 0.99 & 0.84 & 95.3 & 0.97 & 0.98 \\
3 & 0.82 & 0.93 & 91.2 & 0.80 & 0.96 \\
4 & 0.75 & 0.89 & 71.0 & 0.75 & 0.88 \\
5 & 0.66 & 0.91 & 66.9 & 0.66 & 0.85 \\
\hline
\end{tabular}


Figure 2. Confusion matrix of $2,3,4$, and 5 classifications. $\mathrm{AN}=$ pathological voice; $\mathrm{NC}=$ normal voice; $\mathrm{SD}=$ adductor spasmodic dysphonia; PAATOL $=$ unilateral vocal paralysis/vocal atrophy/organic vocal fold lesions; $\mathrm{OL}=$ organic vocal fold lesions; PAAT $=$ unilateral vocal paralysis $/$ vocal atrophy; PA = unilateral vocal paralysis; AT = vocal atrophy.

\section{Two classifications}

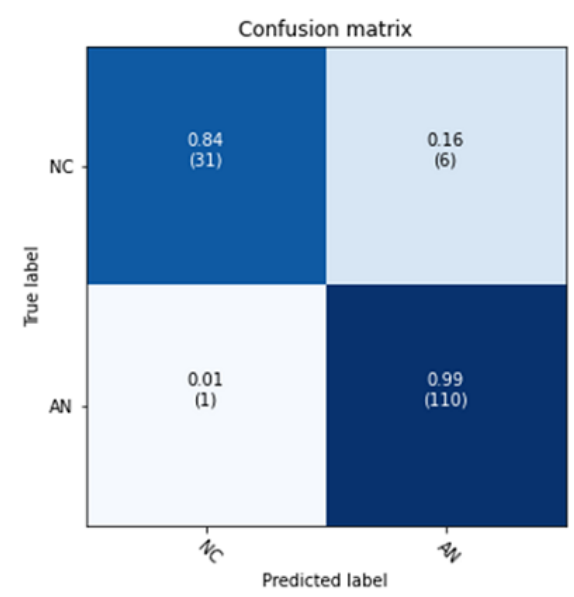

\section{Four classifications}

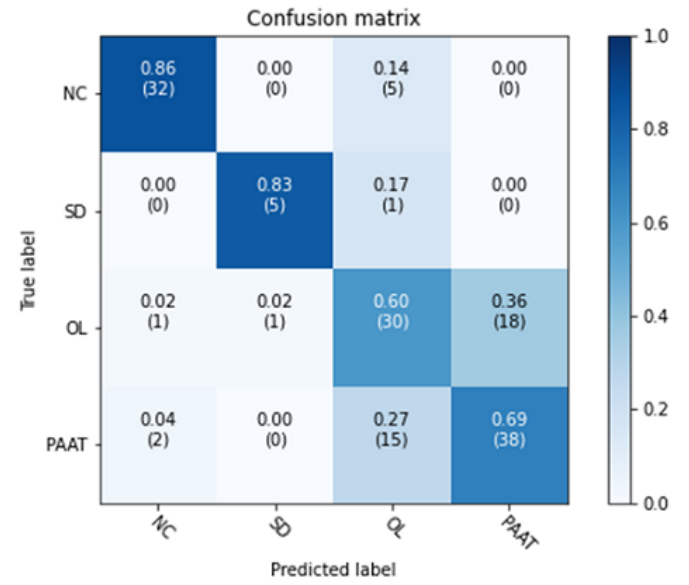

\section{Three classifications}

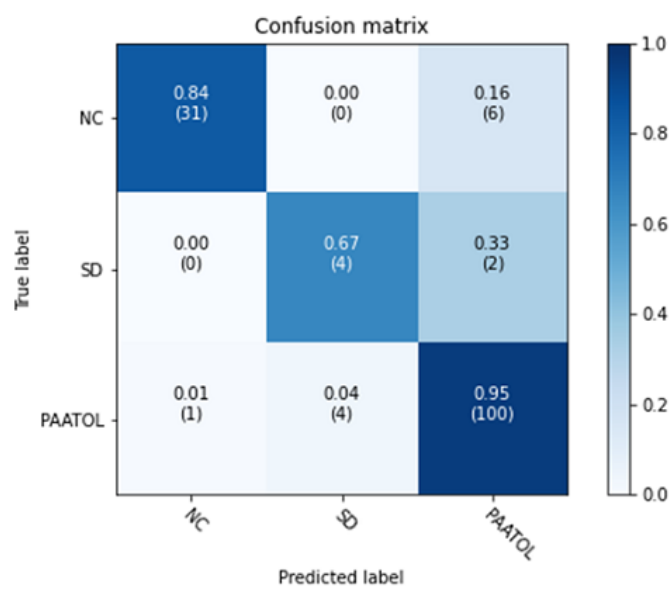

\section{Five classifications}

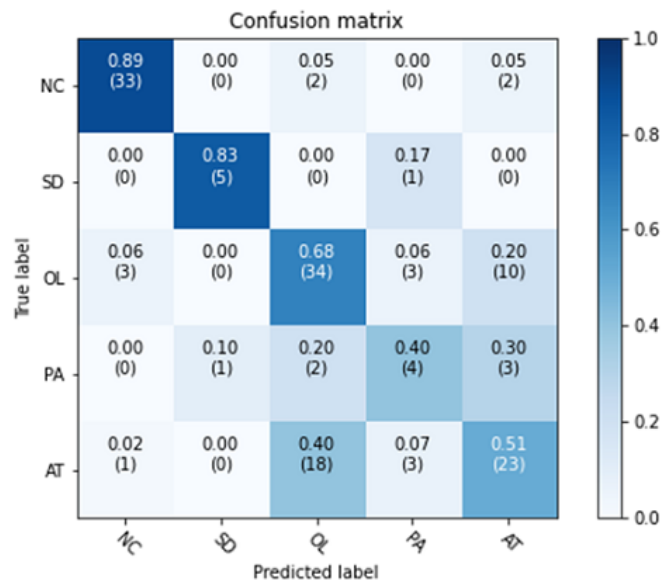


Figure 3. Receiver operating characteristic curves of $2,3,4$, and 5 classifications. NC = normal voice; $\mathrm{SD}=$ adductor spasmodic dysphonia; $\mathrm{PAATOL}$ = unilateral vocal paralysis/vocal atrophy/organic vocal fold lesions; $\mathrm{OL}=$ organic vocal fold lesions; PAAT = unilateral vocal paralysis/vocal atrophy; $\mathrm{PA}=$ unilateral vocal paralysis; $\mathrm{AT}=$ vocal atrophy.

\section{Two classifications}

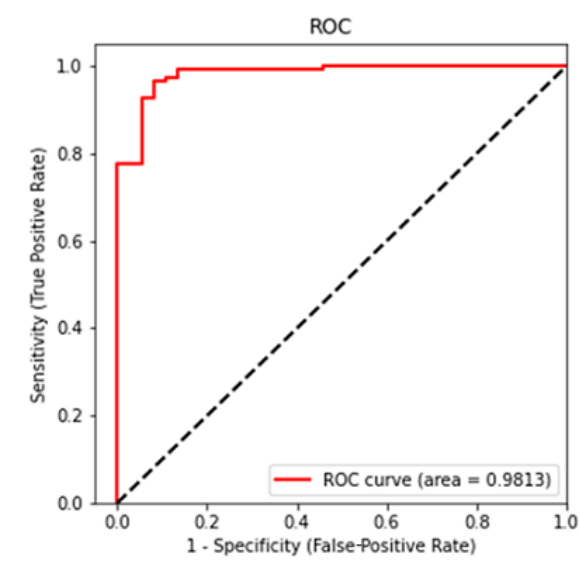

\section{Four classifications}

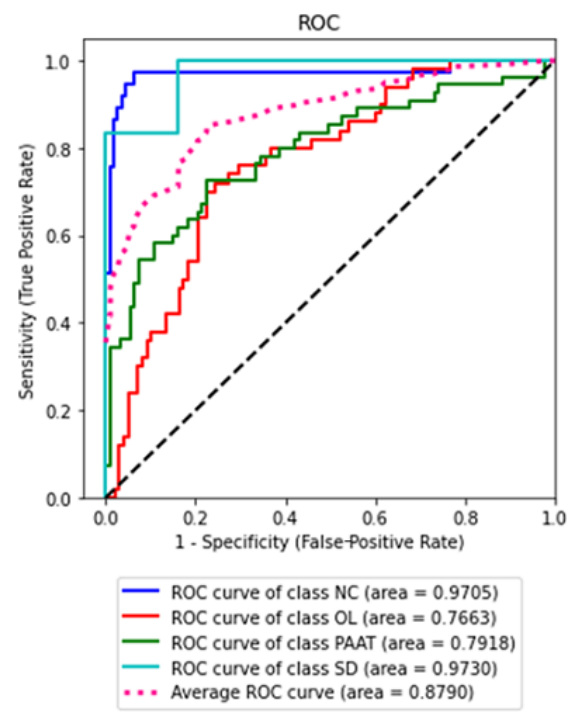

Furthermore, we invited four ENT specialists to identify vocal fold pathology by voice using these 5 classifications. The results are shown in Table 3 and Figure 4. The accuracy rates were

\section{Three classifications}

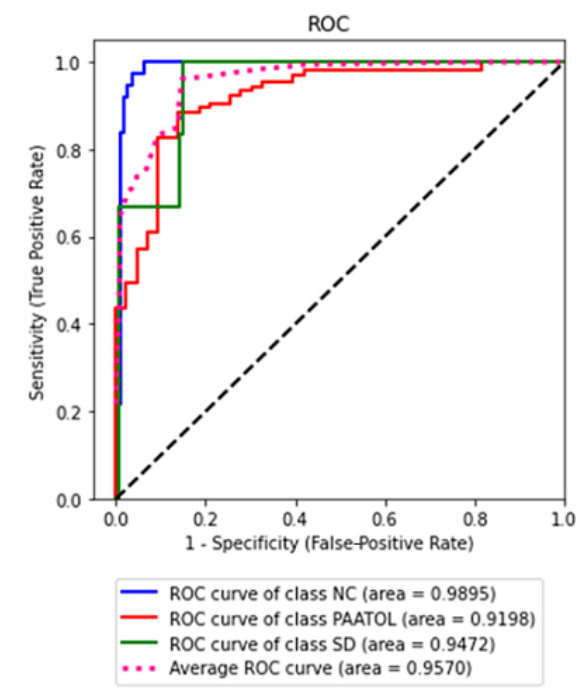

Five classifications

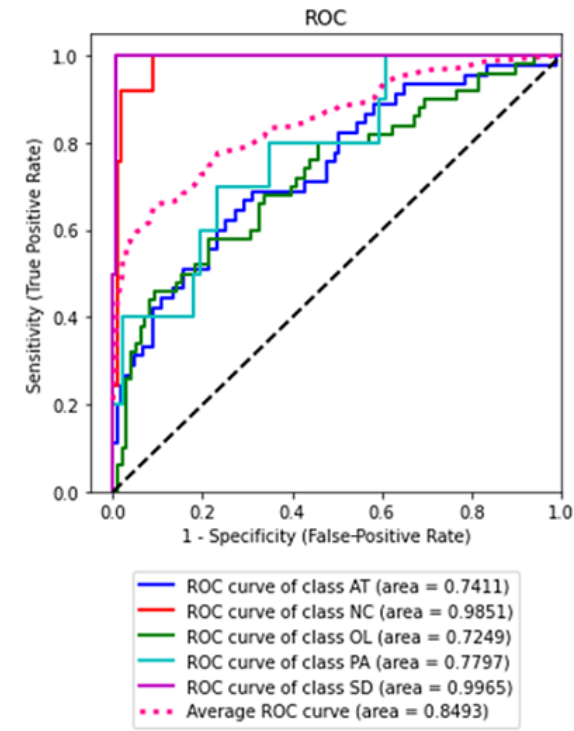

$60.1 \%$ and $56.1 \%$ for the 2 laryngologists and $51.4 \%$ and $43.2 \%$ for the 2 general ENT specialists.

Table 3. Comparison of the performance for a 5-classification condition by our artificial intelligence model and 4 human experts.

\begin{tabular}{llll}
\hline Test participants & Sensitivity & Specificity & Accuracy, $\%$ \\
\hline Deep learning model & 0.66 & 0.91 & 66.9 \\
Laryngologist A (11 years of experience) & 0.61 & 0.89 & 60.1 \\
Laryngologist B (10 years of experience) & 0.63 & 0.88 & 56.1 \\
General ENT ${ }^{\text {a }}$ (8 years of experience) & 0.54 & 0.88 & 51.4 \\
General ENT D (14 years of experience) & 0.42 & 0.85 & 43.2 \\
\hline
\end{tabular}

${ }^{\mathrm{a} E N T}$ : ear, nose, and throat. 
Figure 4. Confusion matrix of 5 classifications in human specialists. $\mathrm{NC}=$ normal voice; $\mathrm{SD}=$ adductor spasmodic dysphonia; $\mathrm{OL}=$ organic vocal fold lesions; PA = unilateral vocal paralysis; AT = vocal atrophy.

\section{Laryngologist A}

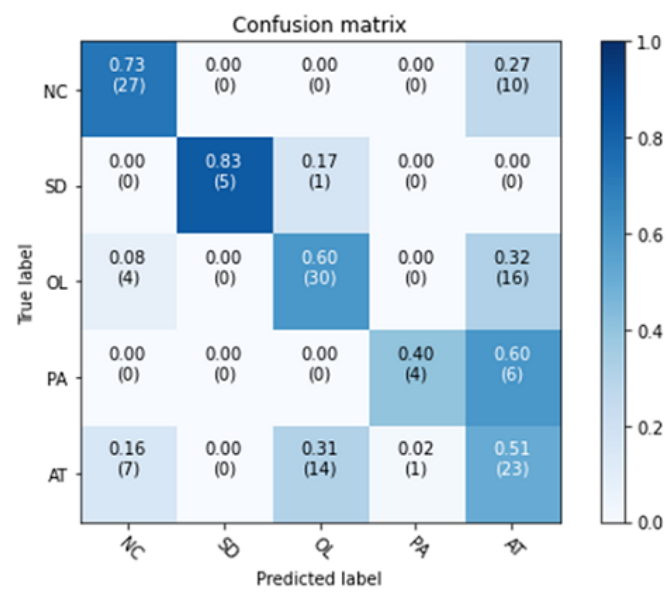

\section{General ENT C}

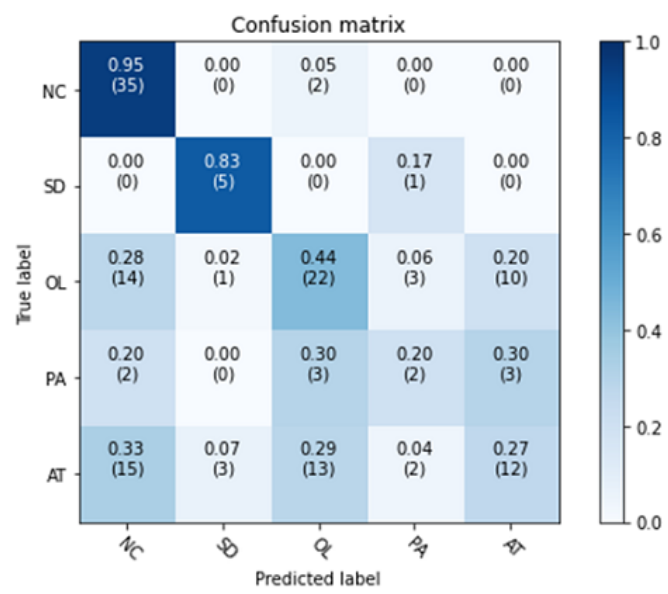

\section{Discussion}

\section{Principal Findings}

During this study, we built a CNN model that could distinguish, with high specificity (91\%), different pathological voices attributable to common vocal diseases based on voice alone. To the best of our knowledge, no previous study has used artificial intelligence to distinguish different types of pathological voices speaking Mandarin. Using our model, we obtained better results by training the $\mathrm{CNN}$ with a whole voice sample than by training it with the vowel sound only or with the essay speech only.

Our model could distinguish normal voice and adductor spasmodic dysphonia with great performance for the 5-classification condition (AUC values: 0.985 and 0.997,

\section{Laryngologist B}

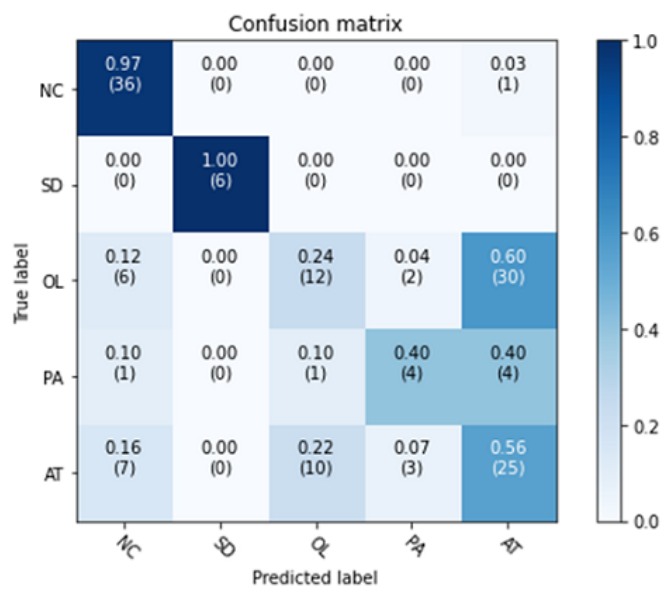

\section{General ENT D}

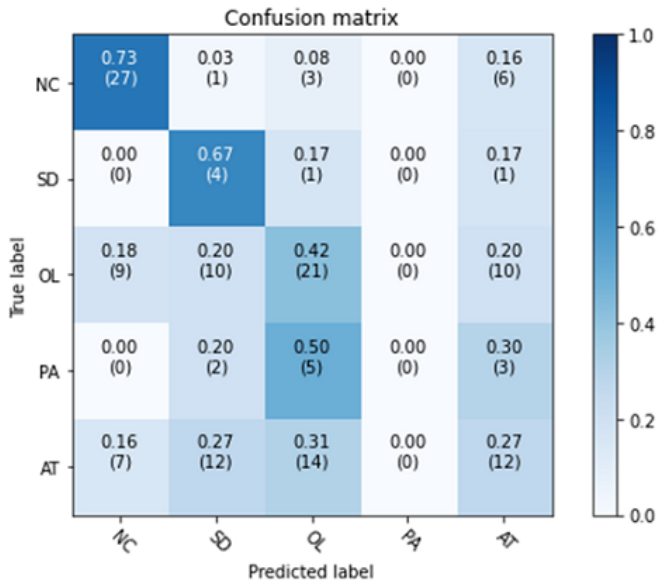

respectively). The overall accuracy of our model was also better than that of all ENT specialists participating in the study. This was compatible with our clinical observation that the first impression of the pathological voice is usually over-ruled by the laryngoscopic examination. Additionally, laryngologists demonstrated higher accuracy when diagnosing voice disorders than general ENT specialists. This may imply that it would be possible to improve the accuracy of human physicians in terms of their impressions of pathological voices by increasing clinical experience. After comparing the accuracy of each classification, we found that artificial intelligence was markedly better than laryngologists when identifying organic vocal fold lesions (artificial intelligence, 68\%; laryngologist A, 60\%; laryngologist B, 24\%). However, laryngologists were slightly better at vocal atrophy identification (artificial intelligence, 51\%; laryngologist A, 51\%; laryngologist B, 56\%). 
Organic vocal fold lesions, unilateral vocal paralysis, and vocal atrophy could result in a closure gap during phonation, inducing a weak and breathy sound [28-30], and vocal fold tension imbalance, inducing diplophonia (when a voice is perceived as being produced with 2 concurrent pitches) [31]. Specifically, in the case of organic vocal fold lesions during vibration, the lesion divided the fold into 2 oscillators. However, in the case of unilateral vocal paralysis, vibrating frequencies were different between the normal vocal fold and paralysis vocal fold. Vocal atrophy will show a breakdown of vibration with a visible repetition in the loss of normal vibration every few glottal cycles [10]. However, the difference in the vibration pattern could only be observed by high-speed video and multislice digital videokymography [10], and the resulting pathological voice is difficult for humans to identify. We speculated that our model may identify related features through deep learning to achieve better outcomes.

Laryngologists could distinguish aged and young patients, and they could validate their judgment during the test based on their knowledge. Vocal atrophy is the most common vocal fold pathology in older patients [3]. Therefore, laryngologists may classify the pathological voice as vocal atrophy if they judged that the voice was that of an aged person.

Regarding misclassification, we have found that our model could successfully identify normal voice and spasmodic dysphonia. However, it was relatively difficult to differentiate organic vocal lesions, unilateral vocal paralysis, and vocal atrophy from each other. Although the vibration patterns were different for these 3 diseases, the different severity levels of disease could result in different degrees of hoarseness. For example, with tiny vocal nodules compared with huge vocal polyps, unilateral vocal paralysis with fair compensation compared with unilateral vocal paralysis with a huge closure gap, and vocal atrophy with a mild anterior closure gap compared with vocal atrophy with a huge closure gap, there could be different degrees of hoarseness in the same group. We assumed that the less severe cases in each group may not show the typical pathological vibration pattern. Further studies are needed to validate our hypothesis.

Four human specialists required 40-80 minutes to identify 148 voice samples of the test set; however, our model only required 30 seconds to perform the same task. The processing time of our model is quite promising in terms of the development of future screening tools.

\section{Comparison With Prior Work}

Most previous studies have used sustained vowel sounds for pathological voice detection $[5,7,8]$. However, other studies have used continuous speech samples for analyses [6]. CNNs extract features automatically from the spectrogram of voice recordings for dysphonia diagnosis, and a larger amount of training data yields better results [32]. Therefore, the CNN used here may have extracted more features from these entire voice samples, thereby achieving better training results with our model.

In this study, we used our voice database for the deep learning approach. The most widely used voice disorder database is the
Massachusetts Eye \& Ear Infirmary (MEEI) Voice Disorders Database (commercially available from KayPENTAX Inc.). The MEEI voice samples (53 normal and 662 pathological voices) are composed of the vowel /ah/ (53 normal and 657 pathological voices) and the utterance of a sentence ("When the sunlight strikes raindrops in the air, they act as a prism and form a rainbow") [33]. However, the voice recordings in the MEEI database were recorded at various sampling rates $(10,25$, and $50 \mathrm{kHz}$ ), and normal and pathological voice recordings were recorded in 2 different environments [32]. Therefore, it was not clear whether artificial intelligence was classifying voice features or environments when trained using the MEEI samples.

The other widely used voice disorder database is the Saarbruecken Voice Database, which contains voice recordings from more than 2000 individuals. Each participant file contains recordings of sustained vowel sounds of $/ \mathrm{a} /, / \mathrm{i} /$, and $/ \mathrm{u} /$ in low, neutral, high, and low-high-low pitches, as well as a continuous speech sentence (“Guten Morgen, wie geht as Ihnen?"). All these samples were recorded at 50-kHz sampling rates and 16-bit resolution [32]. The Saarbruecken Voice Database is considered to be superior to the MEEI database because it uses the same recording environment and the same sampling rates. However, it contains 71 different dysphonia pathologies and many patients recorded in this database had multiple disorders. Therefore, it is difficult to achieve denotation before machine learning.

Our database has several advantages. First, all voice data were from patients visiting our clinics who had detailed chart documents that were carefully reviewed by 2 experienced laryngologists (H-CH and S-YC). Therefore, the quality of the primary data was better than that of the primary data of other studies during which voice data were retrieved from a public database. Second, all voice data were recorded using $44.1-\mathrm{kHz}$ sampling rates and 16-bit resolution, which comprise the standard audio CD format. This widely used format could increase the usability of this data set. Third, we focused on 4 vocal fold diseases that were chosen by experienced laryngologists based on the cause of hoarseness, clinical significance, and prevalence of the disease.

In terms of the cause of hoarseness, adductor spasmodic dysphonia is a focal laryngeal dystonia characterized by irregular and uncontrolled voice breaks that interrupt normal speech [34]. However, organic vocal fold lesions, unilateral vocal paralysis, and vocal atrophy could induce a breathy sound with a different diplophonia pattern [10]. The voice pattern of adductor spasmodic dysphonia is quite different from that of the other pathologies included in this study, and classic cases of adductor spasmodic dysphonia could be diagnosed based on voice alone by experienced laryngologists. The accuracy rates of adductor spasmodic dysphonia among laryngologists using the 5-classification condition were $100 \%$ and $83 \%$ during this study. Therefore, we anticipated that the sensitivity and specificity for diagnosing adductor spasmodic dysphonia could be higher than those of other categories. However, during the first attempt at CNN training, the accuracy of adductor spasmodic dysphonia identification was poor (data not shown). When we attempted to splice the original voice file into 1-second clips while training the model, we found that the voice break in adductor spasmodic dysphonia did not always emerge within every 1-second period. 
After prolonging the duration of the voice clips in the training model, the results improved substantially. This also emphasized that the domain knowledge could significantly influence the training results by tuning the training model according to real clinical conditions.

According to a meta-analysis, in terms of clinical significance, patients with neurologic voice disorders have more challenges than patients with inflammatory or traumatic laryngeal diseases [1]. Specifically, adductor spasmodic dysphonia showed the worst Voice Handicap Index (VHI) score, followed by unilateral vocal paralysis [1]. This result was compatible with our clinical observation that adductor spasmodic dysphonia could markedly interfere with communication and socialization during the daily lives of patients. Although adductor spasmodic dysphonia is a rare disease with a prevalence of 14 out of 100,000 [35], it is worthwhile to offer a model for rapid screening because the symptoms can be treated easily and effectively by regular intralaryngeal botulinum toxin type A injections or surgery [35].

According to VHI scores, unilateral vocal paralysis could also induce a severe voice handicap [1]. The most common cause of unilateral vocal paralysis is an idiopathic or postviral infection, which accounts for $67 \%$ of cases [36]. However, $6 \%$ of patients have underlying malignancies that invade the recurrent laryngeal nerve or vagus nerve [36]. Computed tomography of the skull base, neck, and chest is often recommended during the search for a potential cause of the voice disorder [37]. Thyroid disease, including benign nodules, thyroid malignancy, thyroiditis, hyperthyroidism, and hypothyroidism, may also result in vocal fold paresis [38]. Heman-Ackah et al [38] reported that $47.4 \%$ of patients with unilateral vocal paralysis are diagnosed with concurrent thyroid disease. Therefore, it is important to determine an early diagnosis of unilateral vocal paralysis to investigate the existence of underlying disease.

Organic vocal fold lesions comprise benign lesions, such as nodules, polyps, cysts, polypoid vocal folds, precancerous leukoplakia, and malignant lesions $[39,40]$. The cause of hoarseness with benign and malignant vocal fold lesions involves changes in the laryngeal mucosa and mass effects $[10,40]$. To date, it has been difficult to differentiate organic vocal fold lesions further by voice alone because they involve various pathologies. However, it is worthwhile to inform patients about the possibility of organic vocal fold lesions and to advise them to undergo further investigations. Early stage malignant lesions and benign lesions could be treated with office-based surgery, which is safer and relatively inexpensive compared with surgery in the operating room $[29,41,42]$

The most common cause of vocal atrophy is aging. Aging may result in atrophic musculature and a thinner lamina propria of the vocal fold [43]. However, vocal atrophy can also occur in a relatively young population [28]. It may result from a congenital anomaly or prolonged laryngopharyngeal reflux [44]. The symptoms associated with vocal atrophy are relatively subtle compared with those of other vocal fold diseases [45]. The concern about significant underlying diseases is also reduced with vocal atrophy. However, vocal atrophy is the most common vocal fold pathology among patients older than 65 years [3]. With the aging of the population, vocal atrophy may become a significant geriatric issue in the future.

\section{Limitations}

Our study had some drawbacks. First, all the voice files were recorded in the studio with a silent environment, with sensitive audio-recording technology, and using a certain format. Further studies are needed to validate this approach in different recording environments. Second, all the voice data in this study are from Mandarin speakers. Further studies are needed to compare the results of speakers of different languages. Third, the voice sample numbers of each class were unequal because of the different disease prevalence. We have applied several data augmentation methods to ameliorate the influence of these unequal data. Fraile et al [46] showed that laryngeal pathology detection using voice records based on MFCC and prior differentiation by sex can significantly improve the performance. Fang et al [47] also showed that a deep neural network combining supervectors with medical records could improve pathological voice classifications. Therefore, in the future, we will combine demographic data with voice records to improve our model.

\section{Conclusions}

We demonstrated that voice alone could be used for common vocal fold disease recognition using a deep learning application after training with our Mandarin pathological voice database. Specifically, adductor spasmodic dysphonia, organic vocal fold lesions, unilateral vocal paralysis, and vocal atrophy could be recognized, which could increase the potential of this approach to be more beneficial than simply distinguishing a pathological voice from a normal voice. This approach shows clinical potential for use during general screening of different vocal fold diseases based on voice and could be included in quick evaluations during general health examinations. It could also be used for telemedicine in remote regions that lack laryngoscopy services in primary care units. Overall, it could support physicians during prescreening of cases by allowing for invasive examinations to be performed only for cases involving problems with automatic recognition or listening and for professional analyses of other clinical examination results that reveal doubts about the presence of pathologies.

\section{Acknowledgments}

The authors acknowledge financial support from the Ministry of Science and Technology (MOST 109-2926-I-010-501, MOST 107-2314-B-010-015-MY3, MOST 109-2926-I-010-502, MOST 109-2321-B-010-005, MOST 108-2923-B-010-002-MY3, MOST 109-2823-8-010-003-CV, MOST 109-2622-B-010-006, and MOST 109-2321-B-010-006) and Fu Jen Catholic University Hospital (PL-201908012-V and PL-202008005-V). This work was particularly supported by "Development and Construction Plan" of the School of Medicine, National Yang-Ming University, which is now known as National Yang Ming Chiao Tung University 
(107F-M01-0504), and Aiming for the Top University Plan, a grant from Ministry of Education. Funders did not have any role in the study design, data collection and analysis, the decision to publish, or manuscript preparation.

\section{Conflicts of Interest}

None declared.

\section{Multimedia Appendix 1}

Mandarin passage.

[DOCX File, 12 KB-Multimedia Appendix 1]

\section{References}

1. Cohen SM, Dupont WD, Courey MS. Quality-of-life impact of non-neoplastic voice disorders: a meta-analysis. Ann Otol Rhinol Laryngol 2006 Feb;115(2):128-134. [doi: 10.1177/000348940611500209] [Medline: 16514796]

2. Cohen SM, Kim J, Roy N, Asche C, Courey M. Prevalence and causes of dysphonia in a large treatment-seeking population. Laryngoscope 2012 Feb;122(2):343-348. [doi: 10.1002/lary.22426] [Medline: 22271658]

3. Davids T, Klein AM, Johns MM. Current dysphonia trends in patients over the age of 65: is vocal atrophy becoming more prevalent? Laryngoscope 2012 Feb;122(2):332-335. [doi: 10.1002/lary.22397] [Medline: 22252988]

4. Stachler RJ, Francis DO, Schwartz SR, Damask CC, Digoy GP, Krouse HJ, et al. Clinical Practice Guideline: Hoarseness (Dysphonia) (Update). Otolaryngol Head Neck Surg 2018 Mar;158(1_suppl):S1-S42. [doi: 10.1177/0194599817751030] [Medline: 29494321]

5. Fang SH, Tsao Y, Hsiao MJ, Chen JY, Lai YH, Lin FC, et al. Detection of Pathological Voice Using Cepstrum Vectors: A Deep Learning Approach. J Voice 2019 Sep;33(5):634-641. [doi: 10.1016/j.jvoice.2018.02.003] [Medline: 29567049]

6. Umapathy K, Krishnan S, Parsa V, Jamieson DG. Discrimination of pathological voices using a time-frequency approach. IEEE Trans Biomed Eng 2005 Mar;52(3):421-430. [doi: 10.1109/TBME.2004.842962] [Medline: 15759572]

7. Godino-Llorente JI, Gómez-Vilda P, Blanco-Velasco M. Dimensionality reduction of a pathological voice quality assessment system based on Gaussian mixture models and short-term cepstral parameters. IEEE Trans Biomed Eng 2006 Oct;53(10):1943-1953. [doi: 10.1109/TBME.2006.871883] [Medline: 17019858]

8. Costa S, Neto B, Fechine J. Pathological voice discrimination using cepstral analysis, vector quantization and hidden Markov models. 2008 Presented at: 8th IEEE International Conference on BioInformatics and BioEngineering; October 8-10, 2008; Athens, Greece. [doi: 10.1109/bibe.2008.4696783]

9. Salhi L, Mourad T, Cherif A. Voice disorders identification using multilayer neural network. Int Arab J Inf Technol 2010 Apr;2(7):177-185 [FREE Full text]

10. Woo P. Vibratory Characteristics of Diplophonia Studied by High Speed Video and Vibrogram Analysis. J Voice 2019 Jan;33(1):7-15. [doi: 10.1016/j.jvoice.2017.08.013] [Medline: $\underline{30389188]}$

11. Chen SH. The effects of tones on speaking frequency and intensity ranges in Mandarin and Min dialects. J Acoust Soc Am 2005 May;117(5):3225-3230. [doi: 10.1121/1.1872312] [Medline: 15957789]

12. Zhang H, Cisse M, Dauphin Y, Lopez-Paz D. Mixup: beyond empirical risk minimization. arXiv 2018 Apr [FREE Full $\underline{\text { text] }}$

13. Xu K, Feng D, Mi H, Zhu B, Wang D, Zhang L, et al. Mixup-based acoustic scene classification using multi-channel convolutional neural network. Switzerland: Springer Nature; 2018 Presented at: 19th Pacific-Rim Conference on Multimedia; 2018 Sep 21-22; Hefei, China.

14. Branco P, Torgo L, Ribeiro R. A survey of predictive modelling under imbalanced distributions. arXiv 2015 May [FREE Full text]

15. Logan B. Mel Frequency cepstral coefficients for music modeling. 2000 Presented at: 1st International Symposium on Music Information Retrieval; October 23-25, 2000; Plymouth, MA URL: https://ismir2000.ismir.net/papers/logan_paper. pdf

16. Ravi Kumar KM, Ganesan S. Comparison of multidimensional MFCC feature vectors for objective assessment of stuttered disfluencies. Int J Adv Netw Appl 2011;2(5):854-860 [FREE Full text]

17. Peng N, Chen A, Zhou G, Chen W, Zhang W, Liu J, et al. Environment Sound Classification Based on Visual Multi-Feature Fusion and GRU-AWS. IEEE Access 2020;8:191100-191114. [doi: 10.1109/access.2020.3032226]

18. Hussain M, Bird J, Faria D. A study on CNN transfer learning for image classification. 2018 Presented at: 18th Annual UK Workshop on Computational Intelligence; September 5-7, 2018; Nottingham, UK. [doi: 10.1007/978-3-319-97982-3 16]

19. Franzoni V, Biondi G, Milani A. Emotional sounds of crowds: spectrogram-based analysis using deep learning. Multimed Tools Appl 2020 Aug 17;79:36063-36075. [doi: 10.1007/s11042-020-09428-x]

20. Tan M, Le Q. EfficientNet: Rethinking model scaling for convolutional neural networks. arXiv 2020 Sep [FREE Full text]

21. Hu J, Shen L, Sun G. Squeeze-and-excitation networks. 2018 Presented at: IEEE/CVF Conference on Computer Vision and Pattern Recognition; June 18-23, 2018; Salt Lake City, UT. [doi: 10.1109/cvpr.2018.00745] 
22. Perez L, Wang J. The effectiveness of data augmentation in image classification using deep learning. arXiv 2017 Dec [FREE Full text]

23. Wu H, Gu X. Towards dropout training for convolutional neural networks. Neural Netw 2015 Nov;71:1-10. [doi: 10.1016/j.neunet.2015.07.007] [Medline: 26277608]

24. Lym S, Behroozi A, Wen W, Li G, Kwon Y, Erez M. Mini-batch serialization: CNN training with inter-layer data reuse. arXiv 2018 May [FREE Full text]

25. Huang G, Li Y, Pleiss G, Liu Z, Hopcroft J, Weinberger K. Snapshot ensembles: train 1, get M for free. arXiv 2017 Apr 01 [FREE Full text]

26. Smith L. A disciplined approach to neural network hyper-parameters: Part 1 - learning rate, batch size, momentum, and weight decay. arXiv 2018 Apr 24 [FREE Full text]

27. Kingma D, Ba J. Adam: A method for stochastic optimization. arXiv 2017 Jan 30 [FREE Full text]

28. Hu HC, Hung YT, Lin SY, Tung TH, Chang SY. Office-Based Autologous Fat Injection Laryngoplasty for Glottic Insufficiency in Patients Under 50 Years Old. J Voice 2019 Sep;33(5):747-750. [doi: 10.1016/j.jvoice.2018.03.012] [Medline: 29678440]

29. Hu HC, Hung YT, Lin SY, Chang SY. Office-based Autologous Fat Injection Laryngoplasty for Vocal Process Granuloma. J Voice 2016 Nov;30(6):758.e7-758.e11. [doi: 10.1016/j.jvoice.2015.09.003] [Medline: 26454769]

30. Johns MM, Arviso LC, Ramadan F. Challenges and opportunities in the management of the aging voice. Otolaryngol Head Neck Surg 2011 Jul;145(1):1-6. [doi: 10.1177/0194599811404640] [Medline: 21521897]

31. Hong KH, Kim HK. Diplophonia in unilateral vocal fold paralysis and intracordal cyst. Otolaryngol Head Neck Surg 1999 Dec;121(6):815-819. [doi: 10.1053/hn.1999.v121.a94214] [Medline: 10580244]

32. Wu H, Soraghan J, Lowit A, Di Caterina G. Convolutional Neural Networks for Pathological Voice Detection. Annu Int Conf IEEE Eng Med Biol Soc 2018 Jul;2018:1-4. [doi: 10.1109/EMBC.2018.8513222] [Medline: 30440307]

33. Daoudi K, Bertrac B. On classification between normal and pathological voices using the MEEI-KayPENTAX database: issues and consequences. 2014 Presented at: 15th Annual Conference of the International Speech Communication Association; September 14-18, 2014; Singapore URL: https://www.isca-speech.org/archive/archive_papers/interspeech_2014/i14_0198. pdf

34. Hintze JM, Ludlow CL, Bansberg SF, Adler CH, Lott DG. Spasmodic Dysphonia: A Review. Part 1: Pathogenic Factors. Otolaryngol Head Neck Surg 2017 Oct;157(4):551-557. [doi: 10.1177/0194599817728521] [Medline: 28850801]

35. Whurr R, Lorch M. Review of differential diagnosis and management of spasmodic dysphonia. Curr Opin Otolaryngol Head Neck Surg 2016 Jun;24(3):203-207. [doi: 10.1097/MOO.0000000000000253] [Medline: 26900821]

36. Koufman JA, Postma GN, Cummins MM, Blalock PD. Vocal fold paresis. Otolaryngol Head Neck Surg 2000 Apr;122(4):537-541. [doi: 10.1067/mhn.2000.102574] [Medline: 10740174]

37. Ivey CM. Vocal Fold Paresis. Otolaryngol Clin North Am 2019 Aug;52(4):637-648. [doi: 10.1016/j.otc.2019.03.008] [Medline: 31088695]

38. Heman-Ackah YD, Joglekar SS, Caroline M, Becker C, Kim E, Gupta R, et al. The prevalence of undiagnosed thyroid disease in patients with symptomatic vocal fold paresis. J Voice 2011 Jul;25(4):496-500. [doi: 10.1016/j.jvoice.2010.03.008] [Medline: 20728306]

39. Naunheim MR, Carroll TL. Benign vocal fold lesions: update on nomenclature, cause, diagnosis, and treatment. Curr Opin Otolaryngol Head Neck Surg 2017 Dec;25(6):453-458. [doi: 10.1097/MOO.0000000000000408] [Medline: 29099730]

40. Rzepakowska A, Sielska-Badurek E, Cruz R, Sobol M, Osuch-Wójcikiewicz E, Niemczyk K. Narrow band imaging versus laryngovideostroboscopy in precancerous and malignant vocal fold lesions. Head Neck 2018 May;40(5):927-936. [doi: 10.1002/hed.25047] [Medline: 29322573]

41. Hu HC, Lin SY, Hung YT, Chang SY. Feasibility and Associated Limitations of Office-Based Laryngeal Surgery Using Carbon Dioxide Lasers. JAMA Otolaryngol Head Neck Surg 2017 May 01;143(5):485-491 [FREE Full text] [doi: 10.1001/jamaoto.2016.4129] [Medline: 28208177]

42. Hsu YB, Lan MC, Chang SY. Percutaneous corticosteroid injection for vocal fold polyp. Arch Otolaryngol Head Neck Surg 2009 Aug;135(8):776-780. [doi: 10.1001/archoto.2009.86] [Medline: 19687397]

43. Kendall K. Presbyphonia: a review. Curr Opin Otolaryngol Head Neck Surg 2007 Jun;15(3):137-140. [doi: 10.1097/MOO.0b013e328166794f] [Medline: 17483679]

44. Habesoglu TE, Habesoglu M, Sürmeli M, Deveci I, Toros SZ, Gunes P, et al. Histological changes of rat soft palate with exposure to experimental laryngopharyngeal reflux. Auris Nasus Larynx 2010 Dec;37(6):730-736. [doi: 10.1016/j.anl.2010.03.009] [Medline: 20413235]

45. Gartner-Schmidt J, Rosen C. Treatment success for age-related vocal fold atrophy. Laryngoscope 2011 Mar;121(3):585-589. [doi: 10.1002/lary.21122] [Medline: 21344441]

46. Fraile R, Sáenz-Lechón N, Godino-Llorente JI, Osma-Ruiz V, Fredouille C. Automatic detection of laryngeal pathologies in records of sustained vowels by means of mel-frequency cepstral coefficient parameters and differentiation of patients by sex. Folia Phoniatr Logop 2009;61(3):146-152 [FREE Full text] [doi: 10.1159/000219950] [Medline: 19571549]

47. Fang SH, Wang CT, Chen JY, Tsao Y, Lin FC. Combining acoustic signals and medical records to improve pathological voice classification. APSIPA Transactions on Signal and Information Processing 2019;8(e14):1-11. [doi: 10.1017/atsip.2019.7] 


\section{Abbreviations}

AUC: area under the curve

CNNs: convoluted neural networks

ENT: ear, nose, and throat

MEEI: Massachusetts Eye \& Ear Infirmary

MFCCs: Mel frequency cepstral coefficients

ROC: receiver-operating characteristic

VHI: Voice Handicap Index

Edited by R Kukafka; submitted 25.10.20; peer-reviewed by YB Hsu, V Franzoni; comments to author 09.12.20; revised version received 06.04.21; accepted 25.04.21; published 08.06.21

Please cite as:

Hu HC, Chang SY, Wang CH, Li KJ, Cho HY, Chen YT, Lu CJ, Tsai TP, Lee OKS

Deep Learning Application for Vocal Fold Disease Prediction Through Voice Recognition: Preliminary Development Study

J Med Internet Res 2021;23(6):e25247

URL: https://www.jmir.org/2021/6/e25247

doi: $10.2196 / 25247$

PMID:

(CHao-Chun Hu, Shyue-Yih Chang, Chuen-Heng Wang, Kai-Jun Li, Hsiao-Yun Cho, Yi-Ting Chen, Chang-Jung Lu, Tzu-Pei Tsai, Oscar Kuang-Sheng Lee. Originally published in the Journal of Medical Internet Research (https://www.jmir.org), 08.06.2021. This is an open-access article distributed under the terms of the Creative Commons Attribution License (https://creativecommons.org/licenses/by/4.0/), which permits unrestricted use, distribution, and reproduction in any medium, provided the original work, first published in the Journal of Medical Internet Research, is properly cited. The complete bibliographic information, a link to the original publication on https://www.jmir.org/, as well as this copyright and license information must be included. 